\title{
Giant Bauhin valve adenoma and laparoscopically assisted colonoscopic polypectomy
}

\author{
Saulius Mikalauskas, Rokas Račkauskas, Paulius Žeromskas, Kęstutis Strupas \\ Vilnius University Hospital Santariskiu Clinics, Vilnius, Lithuania
}

Videosurgery Miniinv 2014; 9 (3): 484-485

DOI: $10.5114 /$ wiitm.2014.45045

\begin{abstract}
Nowadays colonoscopic polypectomy has become a routine procedure, which is performed daily. Almost every colorectal benign tumor can be removed endoscopically, although there are some problematic adenomas which are either large in size or difficult to approach. In the past two decades laparoscopically assisted colonoscopic polypectomy has become established in the endoscopic world, which reduced the need of operations for those patients with lesions that are large or hard to approach. The first laparoscopically monitored colonoscopic polypectomy was performed by Franklin in 1991, and reported publicly in 1993. We present a case report of a patient who was diagnosed with Bauhin valve giant adenoma and underwent laparoscopically assisted polypectomy.
\end{abstract}

Key words: colonoscopy, polypectomy, laparoscopically assisted, Bauhin valve adenoma.

\section{Introduction}

Nowadays colonoscopic polypectomy has become a routine procedure, which is performed daily. Almost every colorectal benign tumor can be removed endoscopically, although there are some problematic adenomas which are either large in size or difficult to approach. In the past two decades laparoscopically assisted colonoscopic polypectomy has become established in the endoscopic world, which reduced the need of operations for those patients with lesions that are large or hard to approach [1]. The first laparoscopically monitored colonoscopic polypectomy (LMCP) was performed by Franklin in 1991, and reported publicly in 1993 [2].

We present a case report of a patient who was diagnosed with Bauhin valve giant adenoma and underwent laparoscopically assisted polypectomy.

The aim of this case report is to show that even giant adenomas of the large bowel can be removed using minimally invasive techniques.

\section{Case report}

The patient was in a supine position during the operation. The procedure was performed using an Olympus CF-Q165L colonoscope with diathermal loop for partial resection of adenoma. A colonoscopic needle was used to inject saline and methylene blue. Laparoscopy was performed using an Olympus laparoscope. Pneumoperitoneum was formed using $\mathrm{CO}_{2}$ gas. During laparoscopy abdominal pressure was maintained at $10 \mathrm{~mm} \mathrm{Hg}$. All actions in the peritoneal cavity were performed using soft intestinal graspers.

The patient was admitted for colonoscopic polypectomy because of a previously diagnosed rectal polyp. He had a history of carcinoma of the left colic flexure T4N1MO that penetrated to the jejunum, descending colon and left retroperitoneum, which was treated by extended left hemicolectomy. During the first colonoscopy the endoscopist found a giant $4 \mathrm{~cm}$ $\times 5 \mathrm{~cm}$ circular sessile adenoma of the Bauhin valve

\section{Address for correspondence}

Rokas Račkauskas MD, Vilnius University Hospital Santariskiu Clinics, Erfurto 6-22, 04117 Vilnius, Lithuania, phone: +37060190691,

e-mail: rokas.rackauskas@santa.lt 
with extension into the terminal ileum. During that colonoscopy polypectomy was performed only for 2 other polyps in the ascending colon and rectum. The patient was consulted by abdominal surgeons and endoscopists. The decision to perform laparoscopically assisted colonoscopic polypectomy was agreed on because of existing comorbidities which included terminal renal insufficiency, liver and renal polycystosis, arterial hypertension and ischemic heart disease. Two months later the patient was admitted to hospital again. During this hospitalization the patient underwent the mentioned procedure. The operation was performed under general anesthesia. The endoscopist approached the cecum, and found the Bauhin valve adenoma. First the most prominent masses of the adenoma were removed by thermal loop coagulation. Then the base of the adenoma was lifted by injecting gelofusine solution mixed with methylene blue and then removed with the loop. Next laparoscopy was performed and the terminal ileum was invaginated into the colon; in this way the rest of the adenoma which was extending into the terminal ileum was visualized and was removed with the loop. The site of removed adenoma was cleaned and checked for bleeding and perforation possibility. There were no complications and the operation was finished. The computed tomography (CT) scan after 7 days showed no late perforation or bleeding source. The patient was discharged.

\section{Discussion}

Endoscopic removal of benign colorectal lesions is the gold standard nowadays. Yet there are some polyps that are hard to remove because of their size or their inaccessible growth place [3]. Formerly, those patients would undergo segmental colon resection, but now when minimally invasive surgery is becoming the surgical method of choice even big colorectal polyps can be removed safely. Because of technological progress in medicine, pharmacotherapy and minimally invasive procedures, successful treatment of patients with severe comorbidities can be achieved [4]. As our case report shows, even a $4 \mathrm{~cm} \times 5 \mathrm{~cm}$ circular Bauhin valve adenoma can be removed using minimally invasive methods.

The degree of thermal damage created during polypectomy must balance the need for adequate coagulation of the blood supply feeding the polyp while avoiding damage to the underlying colon wall, which might result in perforation [5]. In our case we performed invagination of the terminal ileum into the ascending colon to access the angle from which we could safely dissect the adenoma from the inner part of the Bauhin valve and terminal ileum. We also performed a CT scan of the abdomen 7 days after the operation to make sure that there was no late perforation of the colon.

\section{Conclusions}

Laparoscopically assisted colonoscopic polypectomy is a safe procedure and should be applied for those patients that are at high risk for open surgery.

\section{References}

1. Prohm P, Weber J, Bönner C. Laparoscopic-assisted coloscopic polypectomy. Dis Colon Rectum 2001; 44: 746-8.

2. Anonymous (1993) SAGES Meeting, Phoenix, AZ, USA, March 31April 3.

3. Ommer A, Limmer J, Mollenberg H, et al. Laparoscopic assisted colonoscopic polypectomy: indications and results. Zentralbl Chir 2003; 128: 195-8.

4. Wierzchowski P, Dabrowiecki S, Szczesny W. Urgent endoscopy in elderly patients with non-variceal upper gastrointestinal bleeding. Videosurgery Miniinv 2012; 7: 246-50.

5. Nelson D. Colonoscopy and polypectomy. Hematol Oncol Clin North Am 2002; 16: 867-74.

Received: 3.12.2013, accepted: 17.02.2014. 